\title{
Psychotherapeutic Treatments for Posttraumatic Stress Disorder
}

\section{Huiping Zhang ${ }^{1,2 \star}$}

${ }^{1}$ Departments of Psychiatry, Yale University School of Medicine, New Haven, CT, USA

${ }^{2}$ VA Connecticut Healthcare System, Department of Psychiatry, West Haven, CT, USA

Posttraumatic stress disorder (PTSD) is a severe anxiety disorder involving intense fear, hopelessness, and horror. PTSD develops after patients experience traumatic events such as actual or threatened death, serious injury, military combat, natural disaster, or sexual violation. Patients with PTSD usually show three main types of symptoms: reexperiencing the traumatic events, avoiding reminders of the trauma, and increased anxiety and emotional arousal. The lifetime prevalence of PTSD is $6.8 \%$, and the 12 -month prevalence is 3.5\% among general adults in the United States [1]. Moreover, patients with PTSD have high rates of comorbid psychiatric disorders (e.g., depressive disorders, anxiety disorders, substance use disorders, and personality disorders, etc) $[2,3]$. The magnitude of the disability and suffering attributable to PTSD has proved a great need for efficient treatment of PTSD and PTSD-associated psychiatric disorders.

The primary treatment methods for PTSD are psychotherapy and medication. Psychotherapy is usually the first choice for PTSD treatment, especially in PTSD-affected children for whom pharmacological treatments are not appropriate. Psychotherapy is often used to treat emotional problems and mental health conditions. Two types of psychotherapy have been applied in PTSD treatment. One is trauma-focused cognitive behavioral therapy (TF-CBT) [4] and another is eye movement desensitization and reprocessing (EMDR) [5]. TF-CBT involves (1) carefully and gradually "exposing" PTSD patients to thought, feelings, and situations that remind them of the trauma, (2) identifying upsetting thoughts about the traumatic event, and (3) helping patients return to healthy states of functionality. EMDR incorporates elements of TF-CBT with eye movements that are thought to "unfreeze" the brain's information processing system, which is inhibited by PTSD. If psychotherapy is ineffective in treating PTSD in adults, antidepressant medications such as paroxetine [6], mirtazapine [7], amitriptyline [8] or phenelzine [9] are sometimes also used while taking into consideration the medicinal side effects. To maximize treatment success, an integrative approach by combining pharmacotherapy and psychotherapy has also been applied in PTSD treatment $[10,11]$.

The current issue of the Journal of Trauma and Treatment is publishing research articles about the effectiveness of PTSD treatment using psychotherapy. EMDR was found to be effective in treating PTSD in children who were victims of a serious car accident. EMDR was also found to be effective in treating PTSD and comorbid disorders such as paranoid, depressive and borderline traits in adults. These studies provide evidence that early intervention with EMDR is most beneficial for patients with PTSD. Another type of psychotherapy called hypnotic abreaction, which is based on the Ego State Theory (EST) [12], was shown to be an effective and durable treatment for PTSD. Additionally, another study analyzed the association between trauma history and juvenile sexual offending, showing that sexual victimization may result in sexually abusive behaviors.

In conclusion, PTSD is a mental illness triggered by major trauma. The high prevalence and economic burden of PTSD provide strong incentives for the science community to develop novel and more effective treatment methods for PTSD. Furthermore, both psychotherapy and medication may need to consider the influence of genetic background of patients on the outcome of PTSD treatment.

\section{References}

1. Kessler RC, Berglund P, Demler O, Jin R, Merikangas KR, et al. (2005) Lifetime prevalence and age-of-onset distributions of DSM-IV disorders in the National Comorbidity Survey Replication. Arch Gen Psychiatry 62: 593-602.

2. Grant DM, Beck JG, Marques L, Palyo SA, Clapp JD (2008) The structure of distress following trauma: posttraumatic stress disorder, major depressive disorder, and generalized anxiety disorder. J Abnorm Psychol 117: 662-672.

3. Pietrzak RH, Goldstein RB, Southwick SM, Grant BF (2011) Prevalence and Axis I comorbidity of full and partial posttraumatic stress disorder in the United States: results from Wave 2 of the National Epidemiologic Survey on Alcohol and Related Conditions. J Anxiety Disord 25: 456-465.

4. Cohen JA, Mannarino AP, Perel JM, Staron V (2007) A pilot randomized controlled trial of combined trauma-focused CBT and sertraline for childhood PTSD symptoms. J Am Acad Child Adolesc Psychiatry 46: 811-819.

5. Bisson JI, Ehlers A, Matthews R, Pilling S, Richards D, et al. (2007) Psychological treatments for chronic post-traumatic stress disorder. Systematic review and meta-analysis. Br J Psychiatry 190: 97-104.

6. Vermetten E, Vythilingam M, Southwick SM, Charney DS, Bremner JD (2003) Long-term treatment with paroxetine increases verbal declarative memory and hippocampal volume in posttraumatic stress disorder. Biol Psychiatry 54: 693702.

7. Lewis JD (2002) Mirtazapine for PTSD nightmares. Am J Psychiatry 159: 1948 1949.

8. Davidson JR, Kudler HS, Saunders WB, Erickson L, Smith RD, et al. (1993) Predicting response to amitriptyline in posttraumatic stress disorder. Am J Psychiatry 150: 1024-1029.

9. Lerer B, Bleich A, Kotler M, Garb R, Hertzberg M, et al. (1987) Posttraumatic stress disorder in Israeli combat veterans. Effect of phenelzine treatment. Arch Gen Psychiatry 44: 976-981.

10. Pratchett LC, Daly K, Bierer LM, Yehuda R (2011) New approaches to combining pharmacotherapy and psychotherapy for posttraumatic stress disorder. Expert Opin Pharmacother 12: 2339-2354.

11. Schneier FR, Neria Y, Pavlicova M, Hembree E, Suh EJ, et al. (2012) Combined prolonged exposure therapy and paroxetine for PTSD related to the World Trade Center attack: a randomized controlled trial. Am J Psychiatry 169: 80-88.

12. Barabasz AF, Barabasz M, Watkins JG (2011) Single-session manualized ego state therapy (EST) for combat stress injury, PTSD, and ASD, part 1: the theory. Int J Clin Exp Hypn 59: 379-391.

*Corresponding author: Huiping Zhang, Departments of Psychiatry, Yale University School of Medicine, New Haven, CT, USA, E-mail: huiping.zhang@yale.edu

Received December 24, 2013; Accepted December 26, 2013; Published December 28, 2013

Citation: Zhang H (2013) Psychotherapeutic Treatments for Posttraumatic Stress Disorder. J Trauma Treat S4: e001. doi:10.4172/2167-1222.S4-e001

Copyright: (c) 2013 Zhang $\mathrm{H}$. This is an open-access article distributed under the terms of the Creative Commons Attribution License, which permits unrestricted use, distribution, and reproduction in any medium, provided the original author and source are credited. 